\title{
THE REGULAR OPEN-OPEN TOPOLOGY FOR FUNCTION SPACES
}

\author{
KATHRYN F. PORTER \\ Department of Mathematical Sciences \\ Saint Mary's College of California \\ Moraga, CA 94575 \\ (Received July 6, 1993 and in revised form March 13, 1995)
}

\begin{abstract}
The regular open-open topology, $T_{\text {roo, }}$, is introduced, its properties for spaces of continuous functions are discussed, and $T_{\text {roo }}$ is compared to $T_{o o}$, the open-open topology. It is then shown that $T_{\text {roo }}$ on $H(X)$, the collection of all self-homeomorphisms on a topological space, $(X, T)$, is equivalent to the topology induced on $H(X)$ by a specific quasi-uniformity on $X$, when $X$ is a semi-regular space.
\end{abstract}

KEY WORDS AND PHRASES. Compact-open topology, admissible topology, open-open topology quasi-uniformity, regular open set, semi-regular space, topological group.

1992 AMS SUBJECT CLASSIFICATION CODES. Primary 54C35, 57S05 Secondary 54H99.

\section{INTRODUCTION.}

A set-set topology is one which is defined as follows: Let $(X, T)$ and $\left(Y, T^{*}\right)$ be topological spaces. Let $\mathrm{U}$ and $\mathbf{V}$ be collections of subsets of $X$ and $Y$, respectively. Let $F \subset Y^{X}$, the collection of all functions from $X$ into $Y$. Define, for $U \in \mathbf{U}$ and $V \in \mathbf{V},(U, V)=\{f \in F: f(U) \subset V\}$. Let $S(\mathbf{U}, \mathbf{V})=\{(U, V): U \in \mathbf{U}$ and $V \in \mathbf{V}\}$. If $S(\mathbf{U}, \mathbf{V})$ is a subbasis for a topology $T(\mathbf{U}, \mathbf{V})$ on $F$ then $T(\mathbf{U}, \mathbf{V})$ is called a set-set topology.

Some of the most commonly discussed set-set topologies are the compact-open topology, $T_{c o}$, which was introduced in 1945 by R. Fox [1], and the point-open topology, $T_{p}$. For $T_{c o}, \mathbf{U}$ is the collection of all compact subsets of $X$ and $\mathbf{V}=T^{*}$, the collection of all open subsets of $Y$, while for $T_{p}, \mathbf{U}$ is the collection of all singletons in $X$ and $\mathbf{V}=T^{*}$.

In section 2 of this paper, we shall introduce and discuss the regular open-open topology for function spaces. It will be shown which of the desirable properties $T_{\text {roo }}$ possesses. In section 3 , Pervin and almost-Pervin spaces are explained.

The fact that $T_{\text {roo }}$, on $H(X)$, is actually equivalent to the regular-Pervin topology of quasiuniform convergence will be discussed in section 4 along with the topic of quasi-uniform convergence. The advantage of the regular open-open topology is the set-set notation which provides us with 
simple notation and, hence, our proofs are more concise than those using the cumbersome notation of the quasi-uniformity.

We assume a basic knowledge of quasi-uniform spaces. An introduction to quasi-uniform spaces may be found in Fletcher and Lindgren's [2] or in Murdeshwar and Naimpally's book [3].

Throughout this paper we shall assume $(X, T)$ and $\left(Y, T^{*}\right)$ are topological spaces.

\section{THE REGULAR OPEN-OPEN TOPOLOGY.}

A subset, $W$, of $X$ is called a regular open set provided $W=\operatorname{Int}_{X}\left(C l_{X}(W)\right)$. If we let $\mathrm{U}$ be the collection of all regular open sets in $X$ and $\mathrm{V}=T^{*}$, then $S_{\text {roo }}=S(\mathrm{U}, \mathrm{V})$ is the subbasis for a topology, $T_{\text {roo, }}$ on any $F \subset Y^{X}$, which is called the regular open-open topology.

A topological space, $X$, is called semi-regular provided that for each $U \in X$ and each $x \in U$ there exists a regular open set, $V$, in $X$, such that $x \in V \subset U$. One can easily show that if $(X, T)$ is a semi-regular space then $T_{\text {roo }} \subset T_{\text {oo }}$, the open-open topology (Porter, [4]) which has as a subbasis the set $S_{o o}=\left\{(U, V): U \in T\right.$ and $\left.V \in T^{*}\right\}$.

We now examine some of the properties of function spaces the regular open-open topology possesses. The first two theorems also hold for the open-open topology even even when $X$ is not semi-regular. The proofs of these two theorems are straightforward and are left to the reader.

THEOREM 1. Let $(X, T)$ be a semi-regular space and $F \subset C(X, Y)$. If $\left(Y, T^{*}\right)$ is $\mathrm{T}_{\mathbf{i}}$ for $\mathrm{i}=$ $0,1,2$, then $\left(F, T_{\text {roo }}\right)$ is $\mathrm{T}_{\mathrm{i}}$ for $\mathrm{i}=0,1,2$.

A topology, $T^{\prime}$, on $F \subset Y^{X}$ is called an admissible (Arens [5]) topology for $F$ provided the evaluation map, E: $\left(F, T^{\prime}\right) \times(X, T) \rightarrow\left(Y, T^{*}\right)$, defined by $E(f, x)=f(x)$, is continuous.

THEOREM 2. If $F \subset C(X, Y)$ and $X$ is semi-regular, then $T_{\text {roo }}$ is admissible for $F$.

Arens also has shown that if $T^{\prime}$ is admissible for $F \subset C(X, Y)$, then $T^{\prime}$ is finer than $T_{c o}$. From this fact and Theorem 2, it follows, as it does for $T_{o o}$, that $T_{c o} \subset T_{\text {roo }}$ when $X$ is semi-regular.

THEOREM 3. The sets of the form $(U, V)$ where both $U$ and $V$ are regular open sets in $X$ form a subbasis for $\left(H(X), T_{\text {roo }}\right)$.

PROOF. Let $(U, V)$ be a subbasic open set in $\left(H(X), T_{\text {roo }}\right)$. i.e., $U$ is a regular open set and $O$ is an open set, not necessarily regular. Let $f \in(U, O)$. Then $f(U) \subset O$, so $f \in(U, f(U)) \subset(U, O)$ and $f(U)$ is a regular open set.

Let $(G, \circ)$ be a group such that $(G, T)$ is a topological space, then $(G, T)$ is a topological group provided the following two maps are continuous. (1) $m: G \times G \rightarrow G$ defined by $m\left(g_{1}, g_{2}\right)=g_{1} \circ g_{2}$ and $\Phi: G \rightarrow G$ defined by $\Phi(g)=g^{-1}$. If only the first map is continuous, then we call $(G, T)$ a quasi-topological group (Murdeshwar and Naimpally [3]).

Note that $H(X)$ with the binary operation o, composition of functions, and identity element $e$, is a group. It is not difficult to show that if $(X, T)$ is a topological space and $G$ is a subgroup of $H(X)$ then $\left(G, T_{o o}\right)$ is a quasi-topological group. However, $\left(G, T_{o o}\right)$ is not always a topological group (Porter, [4]) since $\Phi$ is not always continuous although $m$ is always continuous. But we discover the following about the regular open-open topology. 
THEOREM 4. Let $X$ be a semi-regular space and let $G$ be a subgroup of $H(X)$. Then $\left(G, T_{\text {roo }}\right)$ is a topological group.

PROOF. Let $X$ be a semi-regular space and let $G$ be a subgroup of $H(X)$. Let $(U, V)$ be a subbasic open set in $T_{\text {roo }}$ such that both $U$ and $V$ are regular open sets. Let $(f, g) \in m^{-1}((U, V))$. Then, $f \circ g(U) \subset V$ and $g(U) \subset f^{-1}(V)$. So, $(f, g) \in(g(U), V) \times(U, g(U)) \in T_{\text {roo }} \times T_{\text {roo }}$. But $(g(U), V) \times(U, g(U)) \subset m^{-1}((U, V))$. Thus, $m$ is continuous.

Note that the inverse map $\Phi: G \rightarrow G$ is bijective and that $\Phi^{-1}=\Phi$. Thus, in order to show that $\Phi$ is continuous, it suffices to show that $\Phi$ is an open map. To this end, let $(O, U)$ be a subbasic open set in $T_{\text {roo }}$ where $O$ and $U$ are both regular open sets. Clearly, $\Phi((O, U))=$ $((X \backslash U),(X \backslash O))$ since we are dealing with homeomorphisms. Note that if $C, K$ are regular closed sets then $\operatorname{Int}_{X} C$, Int $_{X} K$ are regular open sets. Thus, since $(X \backslash O),(X \backslash U)$ are regular closed sets, $\operatorname{Int}_{X}(X \backslash U), \operatorname{Int}_{X}(X \backslash O)$ are regular open sets. Again, since $G$ is a set of homeomorphisms, $(X \backslash U, X \backslash O)=\left(\operatorname{Int}_{X}(X \backslash U), \operatorname{Int}_{X}(X \backslash O)\right)$ but this is in $T_{\text {roo. }}$. Therefore, $\Phi(O, U)$ is an open set in $T_{\text {roo }}$. So, $\Phi$ is open and we are done.

\section{PERVIN AND ALMOST-PERVIN SPACES.}

A topological space, $(X, T)$, is called a Pervin space (Fletcher [4]) provided that for each finite collection, $\mathcal{A}$, of open sets in $X$, there exists some $h \in H(X)$ such that $h \neq e$ and $h(U) \subset U$ for all $U \in \mathcal{A}$. A topological space, $(X, T)$ is called almost-Pervin provided that for each finite collection, $\mathcal{A}$, of regular open sets, there exists some $h \in H(X)$ such that $h \neq e$ and $h(O) \subset O$ for all $O \in \mathcal{A}$.

Topologies are rarely interesting if they are the trivial or discrete topology. We have previously shown (Porter, [4]) that $\left(H(X), T_{o o}\right)$ is not discrete if and only if $(X, T)$ is a Pervin space. The situation for $T_{\text {roo }}$ is similar.

THEOREM 5. $\left(H(X), T_{\text {roo }}\right)$ is not discrete if and only if $(X, T)$ is almost-Pervin.

PROOF. First, assume that $(X, T)$ is an almost-Pervin space. Let $W$ be a basic open set in $T_{\text {roo }}$ which contains $e$; i.e. $W=\bigcap_{i=1}^{n}\left(O_{i}, U_{i}\right)$ where. $O_{i} \subset U_{i}$ for each $i=1,2,3, \ldots, n$ and $O_{i}$ and $U_{i}$ are regular open sets in $X .\left\{O_{i}^{i=1}: i=1,2,3, \ldots, n\right\}$ is a finite collection of regular open sets in $X$, and $X$ is an almost-Pervin space, hence, there exists some $h \in H(X)$ such that $h \neq e$ and $h\left(O_{i}\right) \subset O_{i} \subset U_{i}$. So, $h \in W$ and $h \neq e$. Therefore, $\left(H(X), T_{\text {roo }}\right)$ is not a discrete space.

Now assume that $\left(H(X), T_{\text {roo }}\right)$ is not discrete. Let $V$ be a finite collection of regular open sets in $X$. Let $O=\bigcap_{U \in V}(U, U)$. Then, $O$ is a basic open set in $\left(H(X), T_{\text {roo }}\right)$ which is not a discrete space. Hence, there exists $h \in O$ with $h \neq e$. So, $(X, T)$ is almost-Pervin.

The above proof, along with the few needed definitions involving $T_{\text {roo, }}$ is an example of the simplification that the definition of $T_{\text {roo }}$ offers over the quasi-uniform definition and notation.

4. QUASI-UNIFORM CONVERGENCE.

Recall that if $Q$ is a quasi-uniformity on $X$, then the topology, $T_{Q}$, on $X$, which has as its 
neighborhood base at $x, B_{x}=\{U[x]: U \in Q\}$, is called the topology induced by $Q$. The ordered triple $\left(X, Q, T_{Q}\right)$ is called a quasi-uniform space. A topological space, $(X, T)$ is quasi-uniformizable provided there exists a quasi-uniformity, $Q$, such that $T_{Q}=T$. In 1962, Pervin [7] proved that every topological space is quasi-uniformizable by giving the following construction.

Let $(X, T)$ be a topological space. For each $O \in T$, define the set $S_{O}=(O \times O) \cup((X \backslash O) \times X)$. Let $S=\left\{S_{O}: O \in T\right\}$. Then $S$ is a subbasis for a quasi-uniformity, $P$, for $X$, called the Pervin quasi-uniformity and, as is easily shown, $T_{P}=T$.

If we use the same basic structure as above but change the subbasis to $S=\left\{S_{O}: O\right.$ is a regular open set $\}$ then the quasi-uniformity induced will be called the regular-Pervin quasi-uniformity, $R P$.

If $(X, Q)$ is a quasi-uniform space then $Q$ induces a topology on $H(X)$ called the topology of quasi-uniform convergence w.r.t. $Q$, as follows: For each set $U \in Q$, let us define $W(U)=\{(f, g) \in$ $H(X) \times H(X):(f(x), g(x)) \in U$ for all $x \in X\}$. Then, $B(Q)=\{W(U): U \in Q\}$ is a basis for $Q^{*}$, the quasi-uniformity of quasi-uniform convergence w.r.t. $Q$ (Naimpally [8]). Let $T_{Q} \cdot$ denote the topology on $H(X)$ induced by $Q^{*}$. $T_{Q^{*}}$ is called the topology of quasi-uniform convergence w.r.t. $Q^{*}$. If $P$ is the Pervin quasi-uniformity on $X, T_{P}$ is the Pervin topology of quasi-uniform convergence and if $R P$ is the regular-Pervin quasi-uniformity on $X$, then $T_{R P}$ is called the regular-Pervin topology of quasi-uniform convergence, $T_{R P}$.

It has been shown that the open-open topology is equivalent to the Pervin topology of quasiuniform convergence (Porter, [4]). It is also true that the regular open-open topology is equivalent to the regular-Pervin topology of quasi-uniform convergence. The method of two proofs are exactly the same and we leave this one for the reader.

THEOREM 6. Let $(X, T)$ be a topological space and let $G$ be a subgroup of $H(X)$. Then, $T_{\text {roo }}=T_{R P}$ on $G$.

ACKNOWLEDGEMENT. The author would like to thank the Committee for Faculty and Curriculum Development at Saint Mary's College for their financial support.

\section{REFERENCES}

1. FOX, R., On Topologies for Function Spaces, Bull. Amer. Math. Soc., 51 (1945), 429-432.

2. FLETCHER, P. and LINDGREN, W., Quasi-uniform Spaces, Lecture Notes in Pure and Applied Mathematics, 77, Marcel Dekker, 1982.

3. MURDESHWAR, M., and NAIMPALLY, S., Quasi-Uniform Topological Spaces, Noordoff, 1966.

4. PORTER, K., The Open-Open Topology for Function Spaces, Inter. J. Math. and Math. Sci., 12 no. 1 (1993), 111-116.

5. ARENS, R., Topologies for Homeomorphism Groups, Amer. J. Math., 68 (1946), 593-610.

6. FLETCHER, P., Homeomorphism Groups with the Topology of Quasi-uniform Convergence, Arch. Math., $\underline{22}$ (1971), 88-92.

7. PERVIN, W., Quasi-Uniformization of Topological Spaces, Math. Ann. 147 (1962), 316-317.

8. NAIMPALLY, S., Function Spaces of Quasi-Uniform Spaces, Indag. Math., 68 (1965), 768-771. 


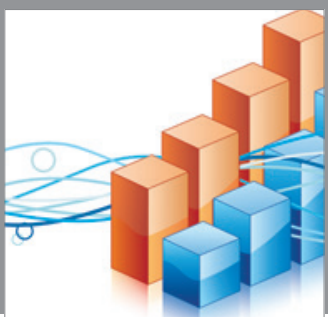

Advances in

Operations Research

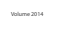

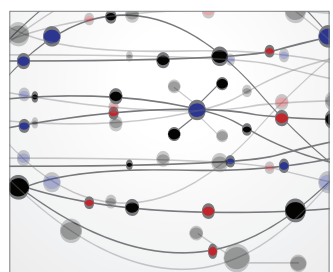

\section{The Scientific} World Journal
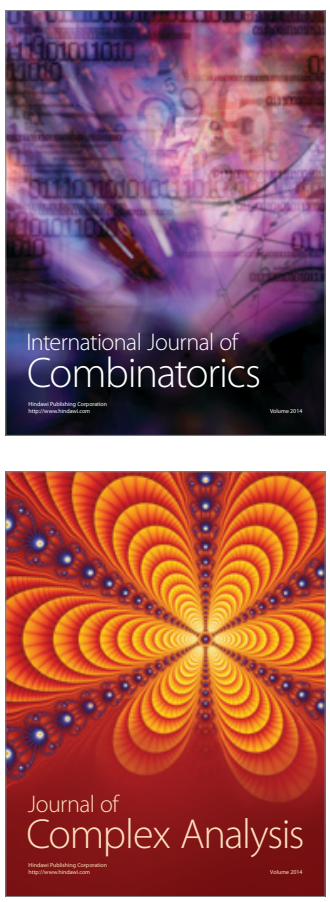

International Journal of

Mathematics and

Mathematical

Sciences
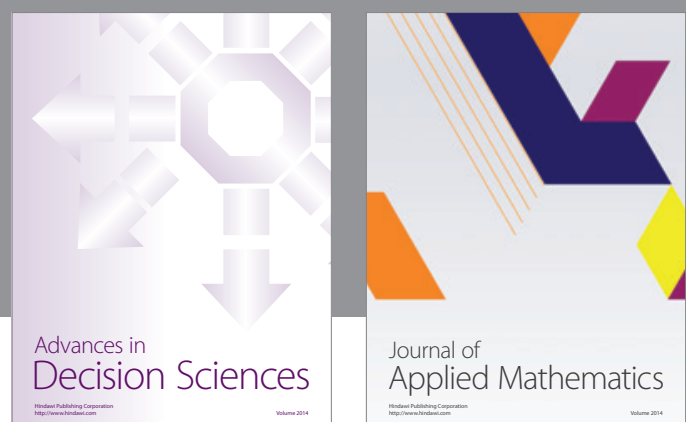

Journal of

Applied Mathematics
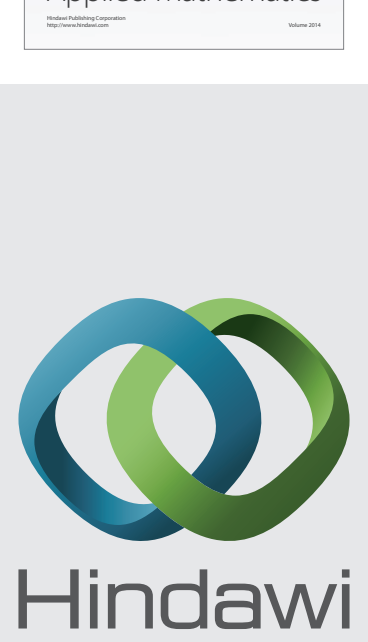

Submit your manuscripts at http://www.hindawi.com
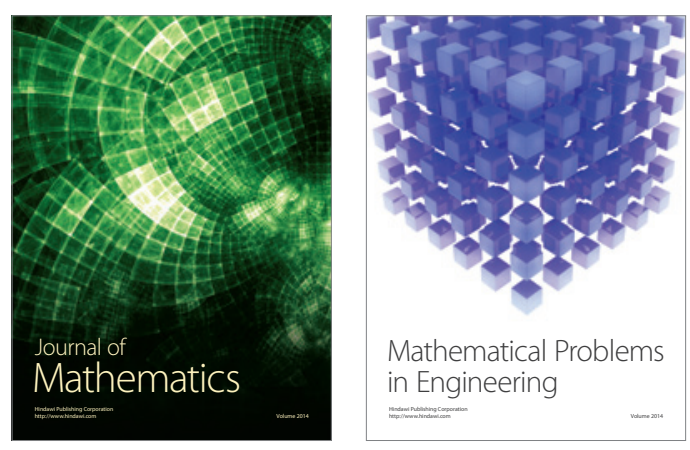

Mathematical Problems in Engineering
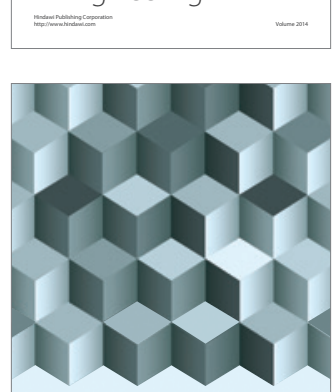

Journal of

Function Spaces
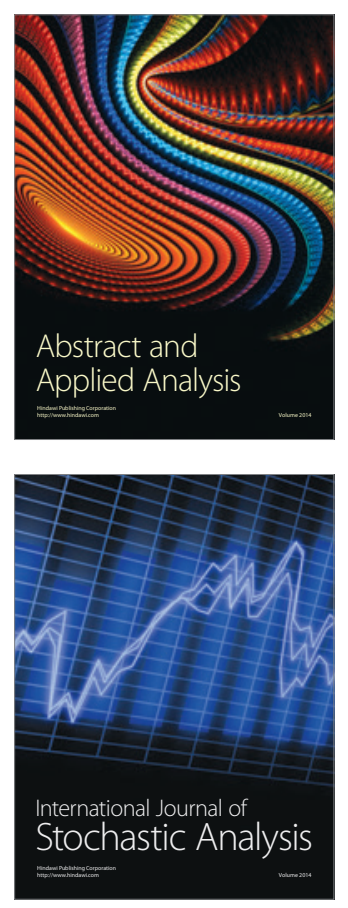

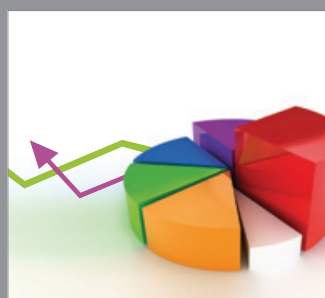

ournal of

Probability and Statistics

Promensencen
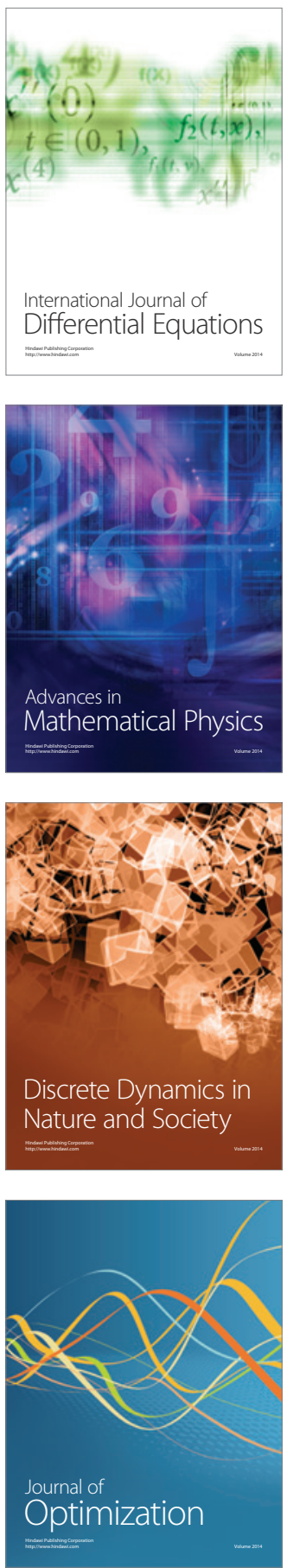